\title{
Modelling the creep anisotropy of nickel-base superalloys with a composite model
}

\section{MÜLLER and M. FELLER-KNIEPMEIER}

Technische Universität Berlin, Institut für Metallforschung, BH18, Strasse des 17. Juni 135, 1000 Berlin 12, Germany

\begin{abstract}
The creep anisotropy of nickel-base superalloys with a cubic $\gamma^{\prime}$ morphology is studied with a composite model. Creep curves and the internal stress distribution in the microstructure under [011] load are calculated with a Finite Element Procedure. Analytical considerations are made for arbitrary loading directions. $\gamma^{\prime}$ cubes (with their interfaces aligned parallel to $\{001\}$ planes) under [001] load have a significant larger creep strength than under [011] or [111] load. Under [011] load the matrix deformation is mainly concentrated in the roof matrix channels.
\end{abstract}

\section{INTRODUCTION}

Modern nickel-base superalloys are strengthened by a high volume fraction of hard $\gamma^{\prime}$ cubes embedded coherently in a soft matrix. The evolution of internal stresses and strains in the microstructure of single-crystal nickel-base alloys during annealing and during creep were measured by different experimental techniques $[1,2,3]$ and calculated using a visco-plastic model in a Finite Element Method (FEM) procedure for the cube and other morphologies under [001] load [4-8]. Previous experimental work has shown that the creep behavior strongly depends on the orientation 
of the $\gamma^{\prime}$ cubes [9-13]. The internal stress distribution of cubes under [011] load and other load directions is studied. Macroscopic creep strains up to $3 \%$ are considered.

\section{MICROMECHANICAL MODEL AND INPUT DATA}

The micromechanical model with its limitations and the FEM (Finite Element Method) procedure are described in [7]. A periodic arrangement of $\gamma^{\prime}$ cubes is simulated. For FEM calculations a volume element containing one eighth of a $\gamma^{\prime}$ precipitate surrounded by its adjacent matrix is chosen (unit cell method) [4-8]. The matrix is permitted to deform both elastically and by creep. A simple creep power law relates the uniaxial equivalent strain rate $\dot{\varepsilon}_{\text {eq }}$ with the equivalent stress $\sigma$ Mises:

$$
\dot{\varepsilon}_{\text {eq }}=A \cdot \sigma_{\text {Mises }}^{\text {n }}
$$

with $\mathbf{n}$ being the creep exponent. The precipitates deform elastically and perfect-plastically according to von Mises yield criterion, as the local stress level reaches the equivalent $\gamma^{\prime}$ yield stress $\sigma_{\text {yield }}^{\gamma^{\prime}}$.

The input data required for the calculations were taken from literature concerning SRR99 or related superalloys at $1123 \mathrm{~K}$. The used data sets in tables 1 and 2 are presented and discussed in [7].

TABLE 1. Elastic Constants and Volume Fraction of $\gamma$ and $\gamma^{\prime}$ Phases

\begin{tabular}{|c|c|c|c|c|}
\hline $1123 \mathrm{~K}$ & Young's modulus E & Shear modulus G & Poisson's ratio $v$ & Volume fraction \\
\hline$\gamma^{\prime}$ Phase & $108 \mathrm{GPa}$ & $98 \mathrm{GPa}$ & 0.40 & $70 \%$ \\
\hline$\gamma$ Phase & $88 \mathrm{GPa}$ & $97 \mathrm{GPa}$ & 0.40 & $30 \%$ \\
\hline
\end{tabular}

TABLE 2. Parameters Characterising the Plastic Behavior of the Two Phases and the Loading Conditions

\begin{tabular}{|c|c|c|c|c|}
\hline $\begin{array}{c}\gamma^{\prime} \text { yield stress } \sigma_{\text {yield }}^{\gamma^{\prime}} \\
\text { in MPa }\end{array}$ & Creep exp. $\mathrm{n}$ & $\begin{array}{c}\mathrm{A} \text { in } \\
\mathrm{Pa}^{-4.7} / \text { normalized } \\
\text { time unit }\end{array}$ & misfit $\delta$ & $\begin{array}{c}\text { External Load } \\
\sigma_{\text {ext in } \mathrm{MPa}}\end{array}$ \\
\hline 650 & 4.7 & $1.43 \cdot 10^{-42}$ & $-0.14 \%$ & 490 \\
\hline
\end{tabular}

The pre-exponential factor A was chosen to be equal for all calculations. As discussed in [7], 100 normalized time units equal 430 hours for the parameterset in tables 1 and 2.

\section{CALCULATIONS}

\subsection{Creep Curves of $\gamma^{\prime}$ Cubes Under [011] Load}

Calculated creep curves for [001] and [011] tensile axis are displayed in Fig.1. The creep strain of cubes under [011] tensile load is found to increase rapidly up to $2 \%$ in [011] direction. No stationary part can be observed. Both creep curves are nearly independent of the amount of coherency stresses. Only the first part of the creep curve of $\gamma^{\prime}$ cubes under [001] is shown for comparison. The whole creep curve of cubes under [001] load and the internal stress distributions for different conditions are presented in [7]. 
3.2 Internal Stress Distribution of $\gamma^{\prime}$ Cubes Under [011] Load

During creep deformation the von Mises stresses in the hard precipitates increase and decrease in the matrix phase (Fig.3). The $\gamma^{\prime}$ phase starts yielding at the point $F$ (see Fig.2) at $t_{\text {norm }} \approx 1$, whereas the center of the cube does not take part in the plastic deformation.

The von Mises stress in the "roof" channels ( $\gamma / \gamma^{\prime}$ interface normal is $45^{\circ}$ off external load axis (see Fig. 2)) decreases rapidly from $570 \mathrm{MPa}$ to $420 \mathrm{MPa}$ and then remains nearly constant from $\mathrm{t}_{\text {norm }} \approx$ 0.2 up to $t_{\text {norm }} \approx 1$ as depicted in Fig. 3. As the $\gamma^{\prime}$ cube undergoes complex deformation for larger elongations, the von Mises stress of the roof channels can further decrease, but remains on a high von Mises stress level of approximately 300MPa. It is obvious that the increase of the von Mises stress level in the center of the cube is coupled to the decrease of the stress level in the roof channel. The vertical matrix channel $\left(\gamma / \gamma^{\prime}\right.$ interface normal is perpendicular to the load axis) rapidly approaches a low stress level of $130 \mathrm{MPa}$ and remains on this low level.

\subsection{Analytical Calculation of the Strain Rate for Cubes under Different Load Directions}

Interfacial edge dislocations act as sources of internal stresses [2,3,7]. Long-range stresses in narrow matrix channels can only be built up parallel to the $\gamma / \gamma^{\prime}$ interfaces $[2,7]$. Shear components of the load tensor, which do not lie in the $\gamma / \gamma^{\prime}$ interface, cannot be compensated by creep-induced stresses in such a way that the von Mises stresss level is further reduced. Under an unaxial load of $\frac{\sigma_{\text {ext }}}{\sqrt{\mathrm{x}^{2}+\mathrm{y}^{2}+\mathrm{z}^{2}}} \cdot[\mathrm{x}, \mathrm{y}, \mathrm{z}]$ the lowest possible von Mises stress for the (100) matrix channel is $\frac{\sigma_{\text {ext }}}{\mathrm{x}^{2}+\mathrm{y}^{2}+\mathrm{z}^{2}} \cdot \sqrt{3 \mathrm{x}^{2} \cdot\left(\mathrm{y}^{2}+\mathrm{z}^{2}\right)}$. The value for the lowest possible von Mises stress for the $(010)$ and (001) matrix channels are given by cyclic permutation of the $x, y$ and $z$ value in the above formula. Table 3 gives the lowest possible von Mises stress, which is expected in the cube matrix channels.

TABLE 3 Lowest Possible von Mises Stress as fraction of $\sigma_{\text {ext }}$ in (100), (010) and (001) Matrix Channels under the External Load Value of $\sigma_{\text {ext }}$ in $[x, y, z]$ Direction.

\begin{tabular}{|l|c|c|c|c|c|c|c|c|}
\hline & $\begin{array}{c}{[001]} \\
\text { Direction }\end{array}$ & {$[012]$} & {$[011]$} & {$[133]$} & {$[111]$} & {$[113]$} & {$[135]$} & {$[123]$} \\
\hline (100) Channel & 0 & 0 & 0 & 0.39 & 0.82 & 0.50 & 0.29 & 0.38 \\
\hline (010) Channel & 0 & 0.69 & 0.87 & 0.86 & 0.82 & 0.50 & 0.76 & 0.78 \\
\hline (001) Channel & 0 & 0.69 & 0.87 & 0.86 & 0.82 & 0.67 & 0.78 & 0.83 \\
\hline
\end{tabular}

FEM calculations have shown that the macroscopic strain rate depends sensitively on the highest von Mises stress level [7]. According to table 3 cubes under load orientations far away from the [001] axis should have significantly higher macroscopic strain rates than under [001] load. Zero von Mises stress in the matrix channel of cubic morphology under [001] load, as predicted in table 3 , can be reached, when the external load $\sigma_{\text {ext }}$ is larger than the product of the $\gamma^{\prime}$ yield stress $\sigma_{\text {yield }}^{\gamma^{\prime}}$ and the $\gamma^{\prime}$ volume fraction (underloading case) [7]. For our parameterset given in tables 1 and 2 (overloading case) the stationary von Mises stress level during creep is $130 \mathrm{MPa}$ for all matrix channels of cubic morphology under [001] load [7]. Our FEM calculations under [011] load (Fig. 3) show that the (001) channel cannot reach zero loading. As the $\gamma^{\prime}$ material starts yielding, the (001) channel is remaining on $130 \mathrm{MPa}$ von Mises stress. For an external load of $490 \mathrm{MPa}$ in [011] direction, the value $0.87 \cdot \sigma_{\text {ext }}$ presented in table 3 for the roof channels is just the plateau value of 420MPa (Fig. 3). The von Mises stress level can only be further reduced from the plateau stress value down to $270 \mathrm{MPa}$ in the roof matrix channels, when the $\gamma^{\prime}$ cube undergoes complex deformation patterns, which produces the compensating shear stresses in the roof channels. Consequently, the matrix stresses in table 3 agree with the FEM result only under the assumption that the $\gamma^{\prime}$ precipitates does not flow during creep deformation. Nevertheless, the values in table 3 
indicate roughly the tendency, how efficiently the internal stress level can be lowered in different orientated matrix channels.

\section{COMPARISON WITH EXPERIMENT}

\subsection{Creep Curves}

Well-formed $\gamma^{\prime}$ cubes under [011] or [111] load, which do not change their shapes during creep at intermediate temperatures between $1033 \mathrm{~K}$ and $1123 \mathrm{~K}$, show a significant higher (average) strain rate than cubes under [001] load [10-13] as predicted by our calculations. It has to be emphasized that we used isotropic yielding conditions for the two phases. The calculated orientation anisotropy of the specimen was only caused by the orientation of the cube faces relative to the external load, whereas other explanations [10-13] are based on the crystallographic anisotropy of the $\gamma$ or $\gamma^{\prime}$ phase.

\subsection{Transmission Electron Microscopy (TEM) Observations}

It was observed [14] that the deformation of a negative misfit alloy (SRR99 at 1033K under 680MPa tensile load) started in the roof matrix channels under [011] tensile load, which can be explained by the higher von Mises stress level in the roof matrix channels compared to the stress level of the vertical channels (Fig. 3). Also, at later stages of creep, the matrix deformation was mainly concentrated in the roof matrix channels, whereas the vertical channels were nearly free of dislocations [14]. This is in accordance with our calculations. The roof matrix channels remain on a high von Mises stress level during the creep deformation (Fig. 3), which locally leads to a large amount of plastic deformation according relation (1).

\section{SUMMARY}

Finite Element calculations with isotropic yielding conditions for the two phases show that the creep behavior of the superalloy composite depends sensitively on the orientation of the $\gamma^{\prime}$ cubes. $\gamma^{\prime}$ cubes (with their interfaces aligned parallel to $\{001\}$ planes) under [001] load have a significant larger creep strength than under [011] or [111] load. Under [011] load the matrix deformation is mainly concentrated in the roof matrix channels.

Acknowledgements:

The authors are indebted to Deutsche Forschungsgemeinschaft for financial support ( $\mathrm{Sfb339}$ ).

\section{REFERENCES}

1. M. V. Nathal, R. A. MacKay and R. G. Garlick, Scripta Met., 22, 1421 (1988)

2. H.A. Kuhn, H. Biermann, T. Ungar and H. Mugrabi, Acta Met. et Mat. $\underline{39}, 2783$ (1991)

3. R. R. Keller, H. J. Maier, H. Renner and H. Mugrabi, Scripta Met. et Mat. 27, 1167 (1992)

4. J. F. Ganghoffer, A. Hazotte, S. Denis and A. Simon, Scripta Met. et Mat. 25, 2491 (1991)

5. T. M. Pollock and A. S. Argon, Acta Met. et Mat. $\underline{40}, 1$ (1992)

6. L. Müller, U. Glatzel and M. Feller-Kniepmeier, Acta Met. et Mat. 40, 1321 (1992)

7. L. Müller, U. Glatzel and M. Feller-Kniepmeier, accepted for publication in Acta Met. et Mat.

8. L. Müller and M. Feller-Kniepmeier, accepted for publication in Scripta Met. et Mat.

9. P. Caron and T. Khan, Mat. Sci. and Eng. 61, 171 (1983)

10. P. Caron, P. J. Henderson, T. Khan and M. McLean, Scripta Met., 20, 875 (1986)

11. R. A. MacKay and R. D. Maier, Met. Trans., 13A , 1747 (1982)

12. P. Caron, T. Khan and Y. G. Nakagawa, Scripta Met. $\underline{20}, 499$ (1986)

13. P. Caron, Y. Ohta, Y.G. Nakagawa, T. Khan, Proc. of the Int. Conf. "Superalloy ' 88 ", SevenSprings (USA), p.285 (1988)

14. T. Kuttner, G. Scheunemann-Frerker and Feller-Kniepmeier, submitted to Phil. Mag. 


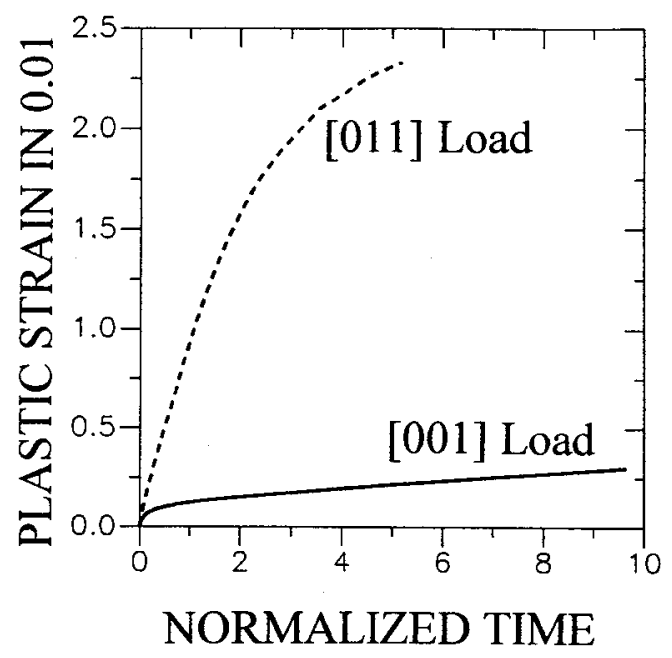

FIG. 1. Evolution of the macroscopic plastic strain in load direction over (normalized) time for $\gamma^{\prime}$ cubes under [001] or [011] external load

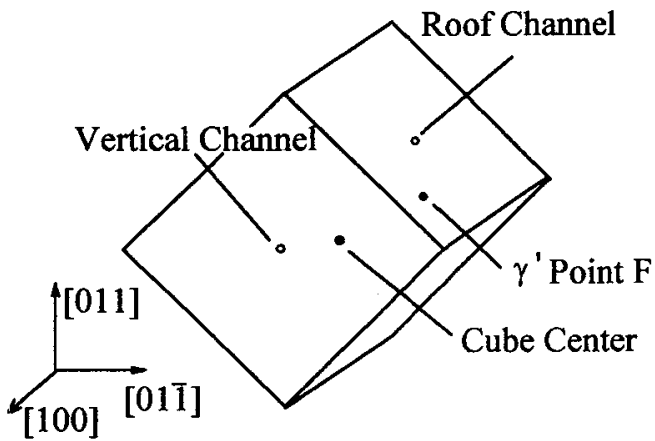

FIG. 2. $\gamma^{\prime}$ cubes under [011] load. Two types of matrix channels, roof channels and vertical channels, can be distinguished.

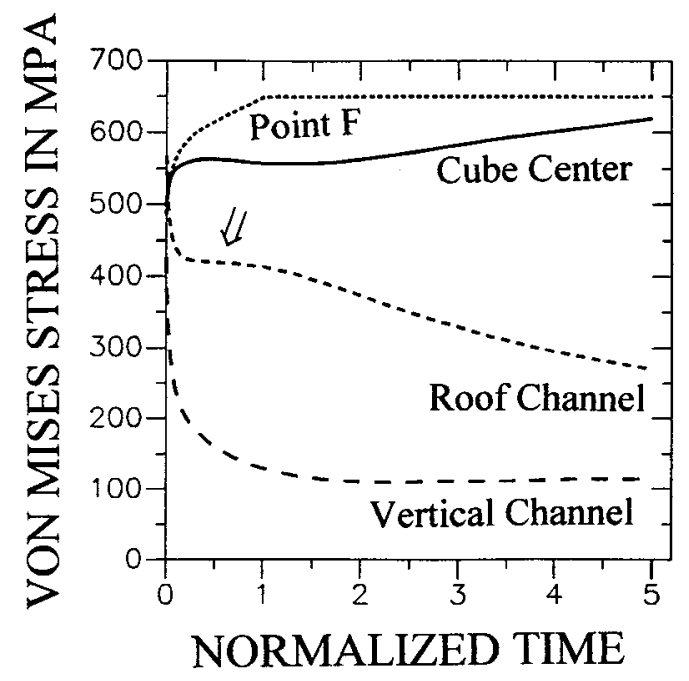

FIG. 3. Evolution of the von Mises stresses in a cubic morphology under $490 \mathrm{MPa}$ [011] load over (normalized) time. The "plateau" of the von Mises stresses in the roof matrix channels is marked by an arrow. 